\title{
Erratum
}

\section{The Hamiltonian Operator Associated with Some Quantum Stochastic Evolutions}

\section{Gregoratti}

Dipartimento di Matematica "F.Brioschi", Politecnico di Milano, piazza Leonardo da Vinci 32, 20133 Milano, Italy. E-mail: matteo.gregoratti@polimi.it

Received: 29 July 2004 / Accepted: 23 January 2006

Published online: 22 March 2006 - C Springer-Verlag 2006

Commun. Math. Phys. 222, 181-200 (2001)

It was kindly pointed out to us by W. von Waldenfels that Section 3.2 of [1] contains an error when the trace operator is introduced for functions in the Sobolev space $H^{\Sigma}\left(\mathbb{R}_{*}^{n} ; \mathfrak{H}\right)$ : we claimed that there exists a bounded operator

$$
\left.\cdot\right|_{\left\{r_{\ell}=s\right\}}: H^{\Sigma}\left(\mathbb{R}_{*}^{n} ; \mathfrak{H}\right) \rightarrow L^{2}\left(\mathbb{R}^{n-1} ; \mathfrak{H}\right)
$$

which naturally defines the trace of each $v$ in $H^{\Sigma}\left(\mathbb{R}_{*}^{n} ; \mathfrak{H}\right)$ as a function $\left.v\right|_{\left\{r_{\ell}=s\right\}}$ in $L^{2}\left(\mathbb{R}^{n-1} ; \mathfrak{H}\right)$, but actually such trace $\left.v\right|_{\left\{r_{\ell}=s\right\}}$ is naturally defined only as a function in $L_{\text {loc }}^{2}\left(\mathbb{R}_{*}^{n-1} ; \mathfrak{H}\right)$ and a trace operator from $H^{\Sigma}\left(\mathbb{R}_{*}^{n} ; \mathfrak{H}\right)$ to $L^{2}\left(\mathbb{R}^{n-1} ; \mathfrak{H}\right)$ can only be closed, with a domain to be specified.

Nevertheless the main result of [1], Theorem 3, is correct and provable through an adjustment of the argument.

We refer to [2] for a detailed introduction of the traces $\left.\cdot\right|_{\left\{r_{\ell}=s\right\}}$ and we list below the points which require an adjustment, that is the points involving $\left.\cdot\right|_{\left\{r_{\ell}=s\right\}}$ which are to be handled taking into account domain constraints.

1. The integration by parts formula (22) needs to be generalized [2] because $\left\langle\left. u\right|_{\partial} Q_{m}\right.$ $\left.|v|_{\partial Q_{m}}\right\rangle_{\mathfrak{H}}$ is not necessarily in $L^{1}\left(\partial Q_{m}\right)$ for every $u$ and $v$ in $H^{\Sigma}\left(\mathbb{R}_{*}^{n} ; \mathfrak{H}\right)$. Therefore, for $\epsilon>0$, we introduce on $\mathbb{R}^{n}$ the totally symmetric indicator function $I_{\epsilon}(r)=\prod_{\ell<\ell^{\prime}}\{1-$ $\left.I_{(-\infty, 0)}\left(r_{\ell} r_{\ell^{\prime}}\right) I_{[0, \epsilon]}\left(\left|r_{\ell}\right|+\left|r_{\ell^{\prime}}\right|\right)\right\}$, which vanishes when $r$ has two small coordinates of opposite sign. Then $I_{\epsilon}(r) \uparrow 1$ as $\epsilon \downarrow 0$ and for every $u$ and $v$ in $H^{\Sigma}\left(\mathbb{R}_{*}^{n} ; \mathfrak{H}\right)$ the following generalized integration by parts formula holds:

$$
\begin{aligned}
\int_{Q_{m}}\left\langle u \mid \sum_{\ell=1}^{n} \partial_{\ell} v\right\rangle_{\mathfrak{H}}= & -\int_{Q_{m}}\left\langle\sum_{\ell=1}^{n} \partial_{\ell} u \mid v\right\rangle_{\mathfrak{H}} \\
& +\lim _{\epsilon \downarrow 0} \int_{\partial Q_{m}}\left(\sum_{\ell=1}^{n} \eta_{m} \cdot e_{\ell}\right)\left\langle\left.\left(I_{\epsilon} u\right)\right|_{\partial Q_{m}}\left|\left(I_{\epsilon} v\right)\right|_{\partial Q_{m}}\right\rangle_{\mathfrak{H}},
\end{aligned}
$$


which reduces to (22), by dominated convergence, every time $\left\langle\left. u\right|_{\partial Q_{m}}|v|_{\partial Q_{m}}\right\rangle_{\mathfrak{H}}$ is in $L^{1}\left(\partial Q_{m}\right)$. This happens if $u$ and $v$ have traces $\left.u\right|_{\partial Q_{m}}$ and $\left.v\right|_{\partial Q_{m}}$ in $L^{2}\left(\partial Q_{m} ; \mathfrak{H}\right)$, or also if, independently of $v,\left.u\right|_{\partial Q_{m}}=\left.\left(I_{\epsilon} u\right)\right|_{\partial Q_{m}}$ for some $\epsilon$.

Analogously, for every $u$ and $v$ in $H_{\text {symm }}^{\Sigma}\left(\left(\mathbb{R}_{*} \times J\right)^{n} ; \mathcal{H}\right)$, the correct version of (23) is the following generalized integration by parts formula [2]:

$$
\begin{aligned}
\left\langle u \mid \sum_{\ell=1}^{n} \partial_{\ell} v\right\rangle_{L^{2}\left((\mathbb{R} \times J)^{n} ; \mathcal{H}\right)}= & -\left\langle\sum_{\ell=1}^{n} \partial_{\ell} u \mid v\right\rangle_{L^{2}\left((\mathbb{R} \times J)^{n} ; \mathcal{H}\right)} \\
& +n \lim _{\epsilon \downarrow 0}\left\{\left\langle\left.\left(I_{\epsilon} u\right)\right|_{\left\{r_{n}=0^{-}\right\}}\left|\left(I_{\epsilon} v\right)\right|_{\left\{r_{n}=0^{-}\right\}}\right\rangle_{\mathfrak{Z} \otimes L^{2}\left((\mathbb{R} \times J)^{n-1} ; \mathcal{H}\right)}\right. \\
& \left.-\left\langle\left.\left(I_{\epsilon} u\right)\right|_{\left\{r_{n}=0^{+}\right\}}\left|\left(I_{\epsilon} v\right)\right|_{\left\{r_{n}=0^{+}\right\}}\right\rangle_{\mathfrak{Z} \otimes L^{2}\left((\mathbb{R} \times J)^{n-1} ; \mathcal{H}\right)}\right\} .
\end{aligned}
$$

2. The unbounded operators $a(s)$ and their domains $\mathcal{V}_{s}$ are to be defined just by Eqs. (32) and (25) of [1], which therefore imply that a vector $\Phi$ in $\mathcal{V}_{s}$ needs to have every single component $\Phi_{n}$ with square integrable trace $\left(\left\|\left.\Phi_{n}\right|_{\left\{r_{n}=s\right\}}\right\|_{\mathfrak{Z} \otimes L^{2}\left((\mathbb{R} \times J)^{n-1} ; \mathcal{H}\right)}<\infty \forall n\right)$.

3. Proposition 3 can still be proved as in [1], but domain constraints for $a\left(0^{-}\right)$and $a\left(0^{+}\right)$are to be dealt with more carefully. Clearly Eq. (36) can always be extended by linearity and it can also be extended by continuity (bounded convergence) to a vector $\Phi$ in $\mathcal{V}_{0^{ \pm}}$every time there is a sequence of vectors $\Phi_{N}$ in $\mathcal{V}_{0^{ \pm}}$satisfying (36) such that $\Phi_{N} \rightarrow \Phi$ in $\mathcal{K}, E \Phi_{N} \rightarrow E \Phi$ in $\mathcal{K}$ and $a(s) \Phi_{N} \rightarrow a(s) \Phi$ in $\mathfrak{Z} \otimes \mathcal{K}$ for $s=0^{-}, 0^{+}$. So the validity of $(36)$ can be extended from $\mathcal{E}\left(H^{1}\left(\mathbb{R}_{*} ; \mathfrak{Z}\right)\right)$ to $n$-particle vectors in $\operatorname{span}\left\{v^{\otimes n} \otimes h \mid v \in H^{1}\left(\mathbb{R}_{*} ; \mathfrak{Z}\right), h \in \mathcal{H}\right\}$ and then to $n$-particle vectors in $H^{1}\left(\mathbb{R}_{*} ; \mathfrak{Z}\right)^{\Im n} \otimes \mathcal{H}$; thanks to Theorem 4 in [2], since the latter space includes $\mathfrak{D}\left(\mathbb{R}_{*}^{n} ; \mathfrak{Z}^{\otimes n} \otimes \mathcal{H}\right) \cap L_{\text {symm }}^{2}\left((\mathbb{R} \times J)^{n-1} ; \mathcal{H}\right)$, Eq. (36) can be extended also to all $n$-particle vectors belonging to $\mathcal{V}_{0^{ \pm}}$and finally to all vectors in $\mathcal{V}_{0^{ \pm}}$.

4. Proposition 6 can still be proved as in [1], even if only the generalized integration by parts formula (23b) is available. The integration by parts formula is applied to prove that $U_{t} \Phi$ belongs to $\mathcal{V}_{0^{-}}$and with (23b) there is a limit w.r.t. $\epsilon \downarrow 0$ which has to be commuted with the integrations in the scalar products. Such operations can be commuted if the vector $\Upsilon$ in $\mathcal{V}_{0}$ is assumed to have components $\Upsilon_{n}$ vanishing in a neighborhood of all the coordinate hyperedges $\left\{r_{j}=r_{\ell}=0\right\}, j \neq \ell$. Then, thanks to Lemma 8 in [2], this class of vectors is large enough to get the thesis.

Acknowledgement. We thank W. von Waldenfels who pointed out the error and G. Gilardi who gave us helpful hints to solve it.

\section{References}

1. Gregoratti, M.: The Hamiltonian Operator Associated with Some Quantum Stochastic Evolutions. Commun. Math. Phys. 222, 181-200 (2001)

2. Gregoratti, M.: Traces of Sobolev functions with one square integrable directional derivative. Math. Meth. Appl. Sci. 29, No. 2 , 157-171 (2006) 